Bio - grafía. Escritos sobre la Biología y su Enseñanza. ISSN 2027-1034

Edición Extraordinaria. p.p. $1296-1313$

Memorias del IX Encuentro Nacional de Experiencias en Enseñanza de la Biología y la

Educación Ambiental. IV Congreso Nacional de Investigación en Enseñanza de la Biología.

\title{
ILUSTRACIÓN CIENTÍFICA COMO POSIBILIDAD DE ENSEÑANZA DE LA BIOLOGÍA EXPERIENCIA DE PRÁCTICA PED AGÓGICA CON ESTUDIANTES DE GRADO 805 DEL INSTITUTO TÉCNICO INDUSTRIAL FRANCISCO JOSÉ DE CALDAS (ITIFJC)
}

\author{
Karen Paola Velandia Candil
}

\section{RESÚMEN}

El presente trabajo exhibe la realización de práctica pedagógica desarrollada dentro del marco del proyecto curricular de Licenciatura en Biología de la Universidad Pedagógica Nacional, durante el periodo 2016 I, encaminado con estudiantes de octavo grado del ITIFJC. El objetivo de este trabajo fue promover la importancia de la ilustración científica en la enseñanza de la biología en la escuela, constituidas en reflexiones en torno a lo que se concibe como la modelización de la enseñanza centrando la discusión en la relación arte-ciencia pensando la ilustración científica como argumento en el contexto educativo.

En la realización de ésta, se tuvo en cuenta sesiones secuenciales dispuestas en seis momentos de análisis Es así, que se resalta el valor de ésta en el campo de la Biología, a propósito de su configuración articulada con la enseñanza de la misma. La aproximación a ello, hace necesario que las estrategias de enseñanza trasciendan lo actitudinal. Es así, donde se problematiza esta implementación para conducirla a una producción real de su importante practica, permitiendo situar dónde y cómo incorporar la ilustración científica como alternativa didáctica en los procesos de enseñanza.

De esta manera, se realiza un ejercicio interesante con los estudiantes de grado octavo del ITIFJC, puntualizando el carácter dominante de la llustración científica en la relación de conocimientos teóricos que permitió examinar la imagen como proceso de conocimiento y de investigación. Además de, reconocer, apreciar y recurrir a la imagen como medio didáctico. Finalmente, se materializa el ejercicio en la representación de organismos tales como artrópodos permitiendo concluir la importancia de la divulgación de esta disciplina en el aprendizaje.

PALABRAS CLAVE: llustración científica, enseñanza de la Biología, habilidades científicas, artrópodos.

\footnotetext{
${ }^{1}$ Estudiante de la Licenciatura en Biología. Grupo de estudios en la Enseñanza de la Biología. Universidad Pedagógica Nacional.Email: paovel.13@gmail.com
} 
Bio - grafía. Escritos sobre la Biología y su Enseñanza. ISSN 2027-1034

Edición Extraordinaria. p.p. $1296-1313$

Memorias del IX Encuentro Nacional de Experiencias en Enseñanza de la Biología y la

Educación Ambiental. IV Congreso Nacional de Investigación en Enseñanza de la Biología.

\begin{abstract}
This work exhibits the realization of pedagogical practice developed within the framework of the curricular project of degree in biology of the National Pedagogical University, during the period 2016 I, directed with eighth grade students of the ITIFJC. The objective of this work was to promote the importance of scientific illustration in the teaching of biology in the school, consisting in reflections around what is conceived as modeling the teaching centering the discussion in the relationship Art-Science thinking the scientific illustration as an argument in the educational context.

in the realization of this, was taken into account sequential sessions arranged in six moments of analysis is thus, which highlights the value of this in the field of biology, with regard to its configuration articulated with the teaching of it. The approximation to this, makes it necessary that the strategies of teaching transcend the attitudinal. Thus, where this implementation is problematizes to lead to a real production of its important practice, allowing to situate where and how to incorporate the scientific illustration as an alternative didactic in the processes of teaching.

In this way, an interesting exercise is carried out with the eighth grade students of the ITIFJC, punctuating the dominant character of the scientific illustration in the relation of theoretical knowledge that allowed examining the image as a process of Knowledge and research. In addition to recognizing, appreciating and resorting to the image as a didactic medium. Finally, it materializes the exercise in the representation of organisms such as arthropods allowing concluding the importance of the dissemination of this discipline in the learning.
\end{abstract}

KEY WORDS: scientific illustration, teaching biology, Scientifics skills,arthropod.

\title{
INTRODUCCIÓN
}

Dentro del Departamento de Biología de la Universidad pedagógica Nacional se reconoce la necesidad de realizar investigaciones "acerca del significado y las implicaciones de ser docente en Biología, en aspectos históricos, epistemológicos, sociológicos, psicológicos, de sus prácticas pedagógicas y de las concepciones en torno a su quehacer." (PCLB, 2011). Pues allí, el desarrollo de la práctica pedagógica sitúa la importancia de la formación en investigación del licenciado en Biología a la hora abordar las situaciones escolares, además de ser un elemento clave la consolidación de su identidad profesional.

El proceso de práctica pedagógica se realiza en el Instituto Técnico Industrial Francisco José de Caldas ubicado en la localidad de Engativa, en el barrio llamado Bosque Popular, dentro de su pilar formativo, cuenta con modalidad técnica. Entre de los principios de formación, se adopta "el desarrollo de pensamiento como insumo estratégico para cultivar las potencialidades de los miembros de la comunidad educativa, comprometiendo además 


\section{Bio - grafía. Escritos sobre la Biología y su Enseñanza. ISSN 2027-1034 \\ Edición Extraordinaria. p.p. $1296-1313$}

Memorias del IX Encuentro Nacional de Experiencias en Enseñanza de la Biología y la

Educación Ambiental. IV Congreso Nacional de Investigación en Enseñanza de la Biología.

el desarrollo de la expresión artística, la creatividad para aplicar en proyectos técnicos y tecnológicos, y en los diferentes campos del conocimiento" ${ }^{2}$, lo que comprendería entonces, la importancia de ofrecer una formación integral y científica, que se orienta al campo de la investigación.

Desde esta perspectiva, el anclaje con la ilustración científica, permite entonces pensar en una nueva configuración de estos elementos educativos, a propósito de la formación artística que se fundamenta en los estudiantes. En tal medida, se teje una relación con el proyecto impulsado por el docente titulado "nuestra Biodiversidad", liderado por un docente titular de área y realizado a la par con estudiantes movidos por el interés del conocimiento local, cabe aclarar que la elección de los organismos parte del interés por el conocimiento y curiosidad que generan y como consigna del proceso, se realizan posters como medio visual de información, empero, se observa que el apoyo visual de estos materiales, se hace a través de la fotografía que en la mayoría de las veces es de la web.

En este sentido, desde la contextualización, se busca en el material didáctico un apoyo en los procesos de aprendizaje alrededor de los organismos locales, a partir de ello, es interesante ver cómo a partir de la ilustración científica se posibilita esa generación, partiendo en primer lugar de la experiencia y del hacer, para la construcción de un conocimiento científico e investigativo por medio del trabajo empírico, y a su vez, también de actividades que impliquen la sensibilización frente a lo que se vivencia y de cómo los estudiantes como sujetos se sitúan.

De esta manera, y con el objetivo de promover la ilustración científica para la enseñanza de un objeto quizá especifico, se plantea entonces el acercamiento a un grupo de organismos tales como los insectos como pilar estratégico en lo que respecta al conocimiento y capacidades desde observación, detalle y fidelidad, para la representación fiel que se acogería como insumo para justificar la importancia de la ilustración cientifica en la enseñanza.

Desde los comienzos de la divulgación y trabajo científico, el ser humano ha gozado de la necesidad de constituir y describir su mundo y la representación del lenguaje visual ha sido una de las primeras formas para hacerlo como se aprecian en las pinturas rupestres. Dentro de las manifestaciones, el arte y la ciencia se vinculan en la ilustración científica, pues acompaña la verbalidad en todo el proceso de surgimiento y adelanto de las Ciencias Naturales.

En la realización del campo de las ciencias Naturales lo que se veía era dibujado para ser estudiado, nombrado y catalogado, lo que significa que la formación del sujeto impera desde tiempos inmemorables, es decir, que para el viajero botánico del siglo xviii, la representación visual era el medio por el cual la habilidad para describir, identificar, clasificar, caracterizar y llegar a la sistematización del conocimiento sobre el mundo

\footnotetext{
${ }^{2}$ Manual de convivencia 2015. Instituto Técnico Industrial Francisco José de Caldas. Artículo 7 Principios de formación.
} 


\title{
Bio - grafía. Escritos sobre la Biología y su Enseñanza. ISSN 2027-1034 \\ Edición Extraordinaria. p.p. $1296-1313$
}

\author{
Memorias del IX Encuentro Nacional de Experiencias en Enseñanza de la Biología y la \\ Educación Ambiental. IV Congreso Nacional de Investigación en Enseñanza de la \\ Biología.
}

natural, era una de las apuestas para la representación visual, medio por el cual la naturaleza se hacia transportable y accesible a los centros de investigación.

Es entonces como la ilustración científica se presenta como alternativa para la realización de investigaciones en torno al estudio profundo de la Biología, es por ello que resulta interesante realizar este tipo de trabajos, pues esto permite el acercamiento, la observación y la descripción detallada, sino que a su vez se configura como un pilar fundamental para la investigación.

Desde luego, reconocer la ilustración científica como una alternativa en el campo de la enseñanza de la Biología, permite potenciar las habilidades científicas a través del uso de ésta en el proceso de aprendizaje y finalmente fortalecer la inclusión de la ilustración científica en la construcción y estudio de la Biología.

La consideración de la ilustración científica como herramienta que acompaña y es vital en el proceso de enseñanza de la Biología, presenta simplificaciones del objeto natural o una especie de realismo aumentado, esto significa que la concepción se hará frente a la teoría en la que está inmerso, teniendo la verbalidad que lo acompaña, que en palabras de Lizárraga Gutiérrez (2004) Lo estético en el caso de la actividad científica, es un correlato o corolario de la verdad. En este sentido, la ilustración científica puede ser definida como aquel proceso que muestra en un gráfico el resultado de una representación que parte de la observación de la realidad y cuya finalidad es su uso por parte de la ciencia.

La llustración Científica realiza una función fundamental en el campo de la ciencia, facilitando la transmisión de conocimiento por parte de investigadores, docentes y diversos profesionales (Hall, Bailey y Tillman, 1997) las ilustraciones científicas podrían cumplir una serie de funciones cognitivas y motivadoras de gran valor en el proceso de aprendizaje.

De esta manera, el desarrollar capacidades de observación y percepción visual, aplicado el análisis e interpretación de organismos, genera habilidades científicas que en concordancia con Sordo (2006) es aprender las habilidades del proceso científico que permite al individuo dominar habilidades como la observación, comunicación, clasificación, medición, deducción y predicción. Así como también resulta ser un saberhacer, que requiere de un proceso cognitivo que haga posible la construcción del conocimiento y por supuesto la acción del individuo que refleje su saber (Ribera, 2008). Resultando entonces en la conjugación de procesos cognitivos entendidos como el proceso del pensamiento científico en la realización de representaciones gráficas.

Dentro de las habilidades integradas del proceso científico, son usadas en conjunto para hacer lo que varios consideran el problema fundamental en la solución de problemas en la ciencia y la experimentación. (Sordo, 2006). Lo que implica, Analizar, observar, aplicar, indagar, que dentro de lo que se contempla en Garzón y Rincón (2012) pueden presentar clasificación desde la acción, es decir, la observación es desde el descubrimiento e identificación de los detalles que son visibles desde la fidelidad de la ilustración, la experimentación, desde el diseño y realización que en este caso vincula el ejercicio 
Bio - grafía. Escritos sobre la Biología y su Enseñanza. ISSN 2027-1034

Edición Extraordinaria. p.p. 1296 - 1313

Memorias del IX Encuentro Nacional de Experiencias en Enseñanza de la Biología y la

Educación Ambiental. IV Congreso Nacional de Investigación en Enseñanza de la Biología.

ilustrativo, apropósito del registro, la comunicación, desde la divulgación y ordenamiento de los resultados. Adicionalmente, de la necesidad por el conocimiento y del quehacer académico científico.

Desde la Didáctica de las Ciencias, el tema de la ilustración científica se sitúa en consideración frente las relaciones entre dibujar-observar, dibujar-recordar, dibujarcomunicar y dibujar- modelizar tal y como lo afirma Márquez (2002); pues es desde allí como empieza a configurarse como un argumento educativo que fortalece el proceso de enseñanza- aprendizaje de los estudiantes, además de dilucidar las apreciaciones estéticas del proceso.

Reconocer que el arte es un instrumento que permite el acceso a saberes que circulan en la escuela es una propuesta posible, desde su potencial sensitivo, que desde su contemplación se enlaza en un conjunto de acciones, de aplicación y de valor de recursos que permiten acceder a un proceso resultante significativo. De esta manera, la enseñanza, aprendizaje de la Biología debe implicar necesariamente el uso de la ilustración científica, pues la construcción del conocimiento también es generado a partir de la observación y de cómo se interpreta y se plasma esa observación, además de ello, de cómo el sujeto desarrolla una serie de habilidades frente a ese sentido estético y/o sensible frente a lo que se configura en su entorno.

De esta manera, la ilustración en el sentido estricto de concebirse, permite develar como los estudiantes pernean un panorama más flexible y dinámico frente a la apreciación de la biodiversidad local, de los organismos en sus ecosistemas, en el caso particular de la entomofauna, a propósito del conocimiento y acercamiento que se configura con este grupo de organismos a través de las representaciones sociales, su elección exclusiva yace desde la posibilidad de concebir la morfología y así mismo su carácter estructural.

Desde luego, se plantea como uno de los mecanismos de aprendizaje para el acercamiento al mundo natural contribuyendo a la creación de aportes desde lo gráfico para la generación de conocimiento, sondeando la representación técnica de la ilustración y su relación con la educación para la promulgación en el interés por el concomiendo de la biodiversidad local y su conservación, además de fortalecer el rigor metódico para su ejecución a través de la representatividad y sentido de la ilustración en el fomento de conocimiento

Siendo así, tomar la ilustración científica como elemento que se instaura en el ejercicio de la representación en el desarrollo artístico, con carácter técnico, permite acentuar en la ciencia biológica, la posibilidad de registrar el entorno natural y con ello contribuir, a su vez, a ampliar las capacidades en los estudiantes en términos de las habilidades científicas tales como la observación, el detalle y el carácter determinante de la cualidad del organismo.

Finalmente, se habla desde el proceso de reflexión que constituye la apreciación del valor de la ilustración científica en la construcción de la enseñanza de la Biología, en los procesos de formación de los estudiantes que a su vez desarrollan al interior de la 


\title{
Bio - grafía. Escritos sobre la Biología y su Enseñanza. ISSN 2027-1034 \\ Edición Extraordinaria. p.p. $1296-1313$
}

\author{
Memorias del IX Encuentro Nacional de Experiencias en Enseñanza de la Biología y la \\ Educación Ambiental. IV Congreso Nacional de Investigación en Enseñanza de la \\ Biología.
}

construcción del campo de conocimiento y de la posibilidad de pesar en la apuesta por la relación estrecha entre el arte y la ciencia como mecanismos que co-dependen para el fruto consolidado dentro y fuera de un contexto en donde se configura, así como también y su procesos de formación en la escuela junto.

\section{MATERIALES Y MÉTODOS:}

La practica pedagógica se enmarco en el paradigma socio-critico, desde los procesos de reflexión crítica de los sujetos participantes, así como de la importancia de la construcción del sujeto frente a su proceso de formación. Con este paradigma se desarrollará el enfoque de investigación cualitativo, que de acuerdo a Colás Bravo et al. (1999) en Morales, A. (2003) fundamenta la acción, la práctica y el cambio, resaltando así su significación en los espacios educativos y sociales, pues promueve la reflexión, la construcción y reconstrucción de la teoría y la practica. Se tiene en cuenta el método Investigación acción participativa, donde se expone que los estudiantes apropian el conocimiento a través del aprender haciendo en una serie de procesos experimentados mediante el uso de los sentidos; con el fin de que tengan la oportunidad de observar, inferir, comprobar, comparar, afianzar, reestructurar, concluir, adquirir y transponer el conocimiento con una perspectiva científica. Por medio de la ilustración científica.

No obstante, se acude a dos modos de estudio, en primer lugar se habla del modo exploratorio donde se identifican las contribuciones que realiza el escenario no convencional en la dinámica de la ciencia en el mismo margen de acción, y en segundo lugar se habla de un modo aplicativo donde se valoran las relaciones que se establecen entre los espacios convencionales y no convencionales de la enseñanza y a su vez en el reconocimiento de este ejercicio como riqueza en la promulgación de los estudios investigativos de la enseñanza de la Biología.

Dentro del cronograma se disponen varias sesiones de índole instrumental conceptual en lo que respecta a dimensiones en términos de proporción, perspectiva, técnicas y uso de dibujo, tipos de representación, modo de realización y carácter exclusivo de una ilustración científica alrededor de preguntas de justificación de porqué, para qué, así como del significado, impacto, que esta rama de las artes genera en la formación del estudiante en el area de las ciencias naturales, aunque no se logro disponer del cronograma debido a las dinámicas del ejercicio en la Institución, se hace la reflexión desde lo que se realiza con los estudiantes en términos de vivencia y experiencia así como del carácter practico del ejercicio.

En el carácter cualitativo, se tiene en cuenta un orden de trabajo, iniciando con una revisión documental de las categorías conceptuales que se instauran en el proyecto, después se realiza la acción explicativa presentado todo una serie esquematizada sobre el transito de la ilustración científica en el desarrollo de las ciencias, lo que ha posibilitado un estudio fuerte en términos de construcción de saber, en consigna del registro de las actividades, reflexiones y discusión colectiva con el grupo de estudiantes, y finalmente se 
Bio - grafía. Escritos sobre la Biología y su Enseñanza. ISSN 2027-1034

Edición Extraordinaria. p.p. $1296-1313$

Memorias del IX Encuentro Nacional de Experiencias en Enseñanza de la Biología y la

Educación Ambiental. IV Congreso Nacional de Investigación en Enseñanza de la Biología.

establecen dinámicas de interacción-observación e ilustración de los organismos así como el registro teórico de las observaciones de grupo y apartados de la experiencia del ejercicio de los estudiantes.

La sistematización del ejercicio se llevará a cabo en el diario de campo que según Bonilla citado en Calero y Conti (2009) "es una invitación a visitar la práctica pedagógica vivida, describir densamente las experiencias y promover la renovación del quehacer educativo en la práctica cotidiana", como instrumento didáctico base de la experiencia, asimismo, contar con referentes en la realización, que permite rescatar apartados de la experiencia. Los estudiantes estarán representados por unidades de información resultando en una categoría de reflexión y pilares de conformación de la misma.

Finalmente concebir la ilustración, material que contara como referente la experiencia y el modo activo de la población y con ello entablar un análisis de datos para su posterior discusión.

\section{RESULTADOS Y DISCUSIÓN}

Debido a procesos que alteraron la planeación de cronograma inicial, se fomentan dos momentos: el primero de ellos es la realización de la ilustración científica teniendo como referente la imagen viva en la memoria, allí la habilidad de observar, detallar y clasificar las majestuosidades del individuo permitirían dar cuenta de un ejercicio interesante que justifica la relación que se establece entre organismos.

Para el desarrollo del análisis con el insumo que se obtuvo, se plantean momentos en los cuáles se realizó la implementaciónn.

\section{La ilustración científica en las ciencias}

A lo largo de la historia natural de las ciencias, el arte ha acudido a la naturaleza para desarrollarse y a su vez se restablecer al servicio de la ciencia, mostrando las particularidades formales de los organismos. pues aquello implica estética y expresión humana acompañando el ejercicio de ilustración como proceso de comunicación y comprensión en la ciencia y su enseñanza. Desde el sujeto, se hace necesario educar la percepción del ojo y coordinar la acción de la mano y la observación consciente o la idea o pensamiento. Con lo cual se lleva todo un proceso de apreciación por parte de quien realiza la representación de su realidad como transcripción de lo que observa. Así mismo, la relación de arte-llustración científica no solo se inscribe, también el arte por composición prácticas estéticas que acogen la variación de las formas de la percepción $y$ de ser del sujeto.

En el primer momento de realización, se hace una presentación donde se resaltan valores relaciones con el sentido de la ilustración frente a la construcción científica del conocimiento, lo esbelto que resulta plasmar y documentar lo natural para comunicar de forma sencilla sin alterar sus caracteres únicos de belleza. Siendo un ejercicio importante 
Bio - grafía. Escritos sobre la Biología y su Enseñanza. ISSN 2027-1034

Edición Extraordinaria. p.p. $1296-1313$

Memorias del IX Encuentro Nacional de Experiencias en Enseñanza de la Biología y la

Educación Ambiental. IV Congreso Nacional de Investigación en Enseñanza de la Biología.

pues de allí se esbozan curiosidades frente a cómo se esta modelizando la enseñanza de la Biología de manera fragmentada en el aislamiento de todos los campos del conocimiento, reflexión que se recoge desde los estudiantes.

Se habla de la importancia del lenguaje visual para la conformación de las ciencias naturales, rescatando la facilidad en términos de relación en la construcción de conocimiento frente a lo que se objeta, de la asociación y significación de ese proceso que se realiza en el acercamiento al conocer del campo.

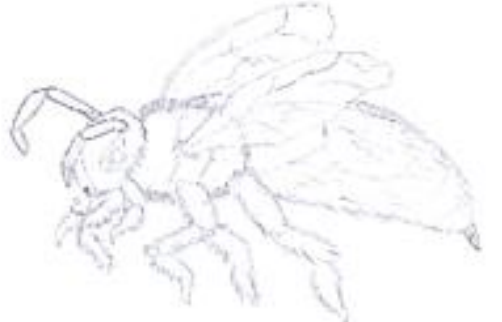

Ilustración 1. Himenóptera. Abeja

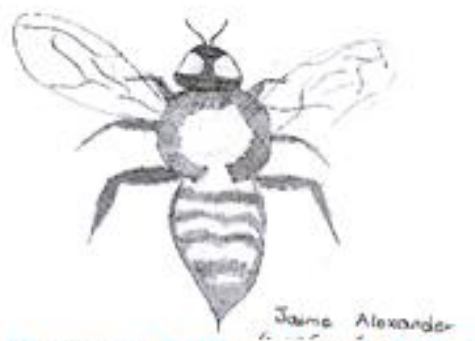

Ilustración 2. Himenóptera. Avispa

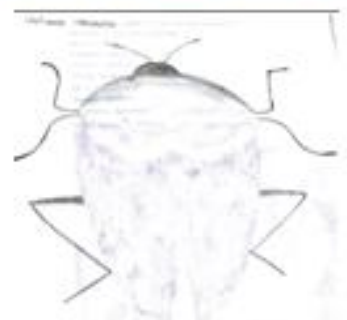

Ilustración 3. Hemíptero. Chinche
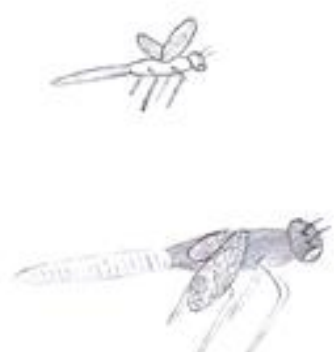

Ilustración 4. Odonatos. Libélula
Sin embargo, se trae a contexto que dentro de las reflexiones constituidas por parte de los estudiantes el carácter de la ilustración científica no se define desde su modalidad, sino desde la apreciación exuberante del profesionalismo y afianzamiento de las ilustraciones por parte de los que las realizan, es así, que dentro de la socialización del impacto de la lustración científica en las ciencias la magnificación de este campo no se exhibe desde el mensaje científico.

\section{Ilustración científica como posibilidad recreada a partir de la memoria}

Las consideraciones frente a lo que se concibe en el ejercicio de ilustrar, requieren de concentración y apreciación del entorno que se desea plasmar e intervenir desde la comprensión del sujeto mismo sin dejar de lado la especificidad del ejercicio. Por tanto, la observación y la acertividad de lo que recorre la imagen en el constructo mental permite confiscar la manera en cómo el sujeto en si mismo reconstruye y originaliza un esquema.

Con los estudiantes, se realizan dos momentos que concretan el resultado en si mismo, haciendo un ejercicio de observación para luego detallar aquello que se ve y que se capta en concentración, se observa que durante el ejercicio de ilustración a partir del recuerdo, los estudiantes denotan dificultad para recrear la imagen, lo que resulta interesante a la hora de observar la ilustración final, cabe aclarar que se toman unas de la totalidad de ilustraciones resultantes debido al criterio que contemplan, sin embargo, las que no se eligen, contemplan otro margen de análisis en términos de transformación desde el concepto y la adecuación antropométrica que se le asigna a la realidad del organismo. 
Bio - grafía. Escritos sobre la Biología y su Enseñanza. ISSN 2027-1034

Edición Extraordinaria. p.p. $1296-1313$

Memorias del IX Encuentro Nacional de Experiencias en Enseñanza de la Biología y la

Educación Ambiental. IV Congreso Nacional de Investigación en Enseñanza de la Biología.

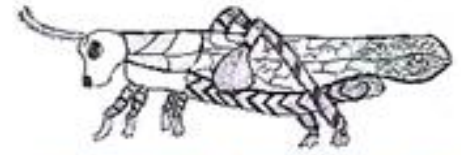

Mustración 5. Orthóptera. Grillo

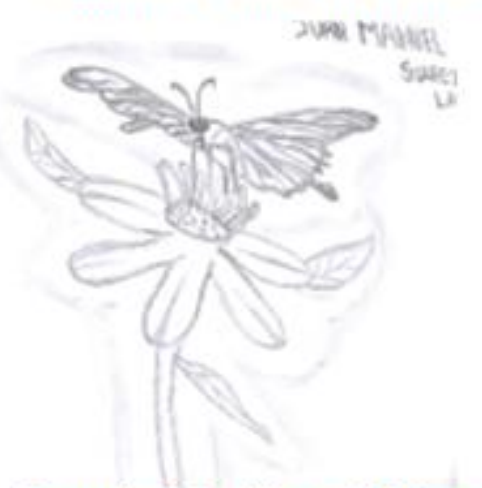

Ilustración 6. Lepidóptera. Mariposa
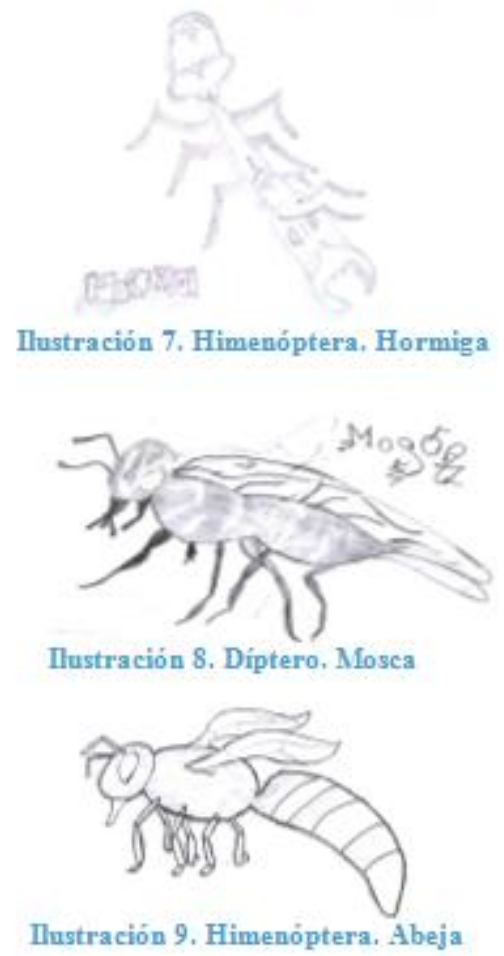

Dentro de la realización del ejercicio, la forma cómo los estudiantes daban cuenta de un producto se plasma en las afirmaciones que en la parte de socialización de la experiencia sustentan (registro cuaderno de campo). Muchos de ellos resaltan el valor útil de la ilustración científica como esa forma sencilla y leal de acercarse a un conocimiento.

El ejercicio práctico de la llustración científica del organismo desde la recreación intima por medio de la memoria, permitió observar en cada una de las ilustraciones de algunos de los estudiantes la proximidad de los caracteres, pues, dentro de la materialización del ejercicio, la intervención del sujeto resulta siendo elemento que configura la realidad del organismo en relación inmediata con las apreciaciones artísticas frente la técnica de la ilustración científica.

Además de resaltarse el valor que presenta y sustenta la ilustración científica en el campo de las ciencias, se detalla potencialidad en tanto se alude a la diferenciación del dibujo a la ilustración propiamente dicha, pues allí se permitió objetar el enfoque y esencia de realización de cada una de las modalidades que se presentan en el lenguaje gráfico, por tanto se devienen de las cualidades únicas que le dan el carácter a este ejercicio, desde el acoplamiento de la realidad natural puro y existente del entorno natural, resaltando d ntegran aquel entorno por supuesto de lo esbelto.etalles que le son propios de diferenciación y caracterización de los organismos que i

De esta manera, se permite analizar que dentro de las ilustraciones realizadas por los estudiantes, se rescatan elementos diferenciales desde el hiperrealismo natural que, en palabras de Cespedosa (2009) este elemento alude al realismo y fidelidad que presenta la ilustración respecto al elemento observado. Así mismo, desde la forma en que los estudiantes va a aproximar las relaciones en el entorno inmediato del organismo, donde no solo se incluye esto anterior, sino que también se ve un ejercicio involucrado desde la emoción y lo que conlleva a un constructo de pensamiento que le permite al estudiante vincular su experiencia generando una perspectiva frente a aquello que acerca, observa y comprende. 


\title{
Bio - grafía. Escritos sobre la Biología y su Enseñanza. ISSN 2027-1034 \\ Edición Extraordinaria. p.p. $1296-1313$
}

\author{
Memorias del IX Encuentro Nacional de Experiencias en Enseñanza de la Biología y la \\ Educación Ambiental. IV Congreso Nacional de Investigación en Enseñanza de la \\ Biología.
}

En concordancia, se habla también de una significación natural de los organismos, lo que alude a aquella designación que se exhibe en la importancia de la ilustración de un elemento $u$ otro en el mismo escenario presentando mayor rigurosidad y detalle como resultado de un acto de percepción mas definitiva y sobresaliente para el estudiante. Sin embrago, también se resalta la fiabilidad del carácter comportamental o de acción del organismo, pues se detalla el valor descriptivo.

\section{"cada detalle, cada línea, cada sombra, luz o difuminado, puestos en sus dibujos expresan con intensión, percepciones que se encontraban de forma pasiva o inutilizadas, pero ahora más elaboradas y complejas" (Lowelfeld, 1986: 9).}

Es importante reconocer que dentro del valor de a ilustración científica no solo se representa una puesta del organismo sino que se busca ilustrar todo aquello que signifique la obra, por tanto, dentro de lo que ya se ha mencionado, el carácter técnico que devela la habilidad de los estudiantes dan cuenta de un sentido de precisión en términos de estructura lo que refiere entonces a una modificación de la expresión en tanto exige conocimiento y desarrollo de destrezas, considerando el reconocimiento de la pertinencia de algunos de los detalle que no serían de fácil acceso visual, es decir, que el conocimiento del ilustrador denota la complejidad en términos de exhibición de elementos clave que pertenecen.

Evocar y trasladar a la mente la visualización del organismo frente a las ideas que él mismo objeta permite vislumbrar las relaciones que el sujeto teje con su contexto en términos de la relación inmediata y de cómo esta construyendo conceptualmente las imágenes con bases teóricas de asociación, determinan su concomiendo, que en palabras de Cocucci (2000) no es mas que el ejercicio del ilustrador desde lo que sabe, mas no desde lo que observa.

No obstante, se habla de la relación sujeto-conocimiento-contexto, también se dilucida dentro de las ilustraciones de los estudiantes que resulta imparcial los elementos que coexisten en la misma ilustración, pues resaltan el mismo detalle en los elementos lo que se da en comprensión pero que también resulta falto de realismo.

En este sentido, es posible objetar que dentro del ejercicio de realización evocando la memoria, resulta imperante comprender las apreciaciones en términos de asociación con lo cercano al sujeto, si bien, éste elemento dará cuenta de la forma en la que la representación visual establezca aspectos de análisis y complejidad de relaciones desde su constructo mental, es así, que la ilustración científica no solo se sustenta por la misma sino que va a permitir formalizar y afianzar todo un proceso complejo e interesante en el proceso de formación y acercamiento al estudio disciplinar de las ciencias, revelando el real sentido e importancia que denotaría este criterio.

Es importante resaltar, que con el grupo de estudiantes no se realizaron sesiones dispuestas a técnicas, modelización y forma de representación de la ilustración científica, por tanto, esto da cuenta de la habilidad de asociar relaciones en el proceso de aprendizaje y de construcciones de saber. 
Bio - grafía. Escritos sobre la Biología y su Enseñanza. ISSN 2027-1034

Edición Extraordinaria. p.p. $1296-1313$

Memorias del IX Encuentro Nacional de Experiencias en Enseñanza de la Biología y la

Educación Ambiental. IV Congreso Nacional de Investigación en Enseñanza de la Biología.

\section{Ilustración científica con carácter antropomórfico}

El resultado de una ilustración que refiera el carácter simbólico de la representación humana, es una constante que se halla dentro del enfrentamiento a la hora de ilustra, si bien, otorgar caracteres antropométricos a un organismo en una ilustración evidencia en tal medida el desconocimiento parcial o total del organismo, pues, dentro de las ilustraciones de los estudiantes, se evidencia que los caracteres diferenciales hacen de la ilustración una muestra viva de anegación de las características propias de los organismos que en palabras de Rogel \& Agudelo (2000) se personifican desde las representaciones sociales que se consideran más favorables al momento de reconocer un organismo diferente al humano.

\section{Huerta: Espacio para la llustración científica}

Concebir distintos contextos para la proximidad real con el entono y con ello la pureza de la ilustración científica, se toma el espacio de la huerta escolar de la institución como escenario que posibilite una buena y provecha gama de ilustración científica, pues, no hay más fidelidad que vivenciar el entorno in situ. Ésta seria la actividad que continuaría con el proceso de reflexión frente al valor de la ilustración científica en estos espacios de formación.

Los estudiantes realizan una recolecta en la huerta, si bien, se menciona que solo se realiza ilustración de insectos, sin embargo, por las dinámicas que se vinieron presentando en el establecimiento, se opta por aprovechar la diversidad ya no de insectos sino de artrópodos como tal para el ejercicio de la ilustración.

Durante el ejercicio, varios de los estudiantes mostraban particular interés y emoción en el ejercicio de recolecta de los Imágen1. Estudiantes ilustrando en la Huerta. Velandia (2016) organismos, sin embargo durante el ejercicio de la ilustración no mostraban mayor destreza en términos de disposición para su realización, esto se debió a que la mayoría de ellos no les motivaba realizar el ejercicio pues, desde sus argumentaciones se encontraba que la afinidad con la actividad de ilustrar era lejana por tanto la consideración del trabajo realizado, además de constituir un esfuerzo mayor en la parte de comprensión y análisis de un organismo real .

Este ultimo aparatado, no solo da cuenta de la extrañeza y distancia de los estudiantes frente al ejercicio de ilustrar asociado al no tener capacidades para hacerlo, pues, dentro de la revisión de antecedentes, este resulta ser un problema común en la comunidad 
Bio - grafía. Escritos sobre la Biología y su Enseñanza. ISSN 2027-1034

Edición Extraordinaria. p.p. $1296-1313$

Memorias del IX Encuentro Nacional de Experiencias en Enseñanza de la Biología y la

Educación Ambiental. IV Congreso Nacional de Investigación en Enseñanza de la Biología.

científica a propósito del estudio de la entomofauna, pues se dice que se debe al no manejo de las técnicas, cuando, desde el ejercicio que se realiza es posible pensar en el potencial y fortalecimiento de habilidades de observación, detalle, proporción para resultar en una sublime apuesta en el conocimiento de los organismos, no solo a ello, sino también del bagaje en conocimiento de las ciencias.

Este espacio de trabajo, permite recrear desde la contemplación de la historia natural el proceso mediante el cual la ilustración científica ha sido protagonista en el desarrollo de ésta. Si bien, permite dimensionar en el estudiante el reto y capacidad del ilustrador científico frente lo natural y cómo a partir de ello se consagrara todo una serie de resultados que luego serian quienes dieron cuenta de un conocimiento especifico del campo disciplinar de la Biología. Adicionalmente, la apuesta desde la transformación de los imaginarios únicos para entender el campo de conocimiento como unidireccional. Pues no solo se genera en el ejercicio sino que desde los estudiantes, la transformación de idealizar y subjetivar el mundo deviene de nuevas perspectivas de movilidad, desde la cognición en términos de conocimientos, afectivos en relación a la preferencia, emocionalidad y expresión estética frente aquello y por supuesto cambio conductual, pues ya se aprecia lo vivo y la vida en sus magnificaciones .

Algunas de las ilustraciones en campo...

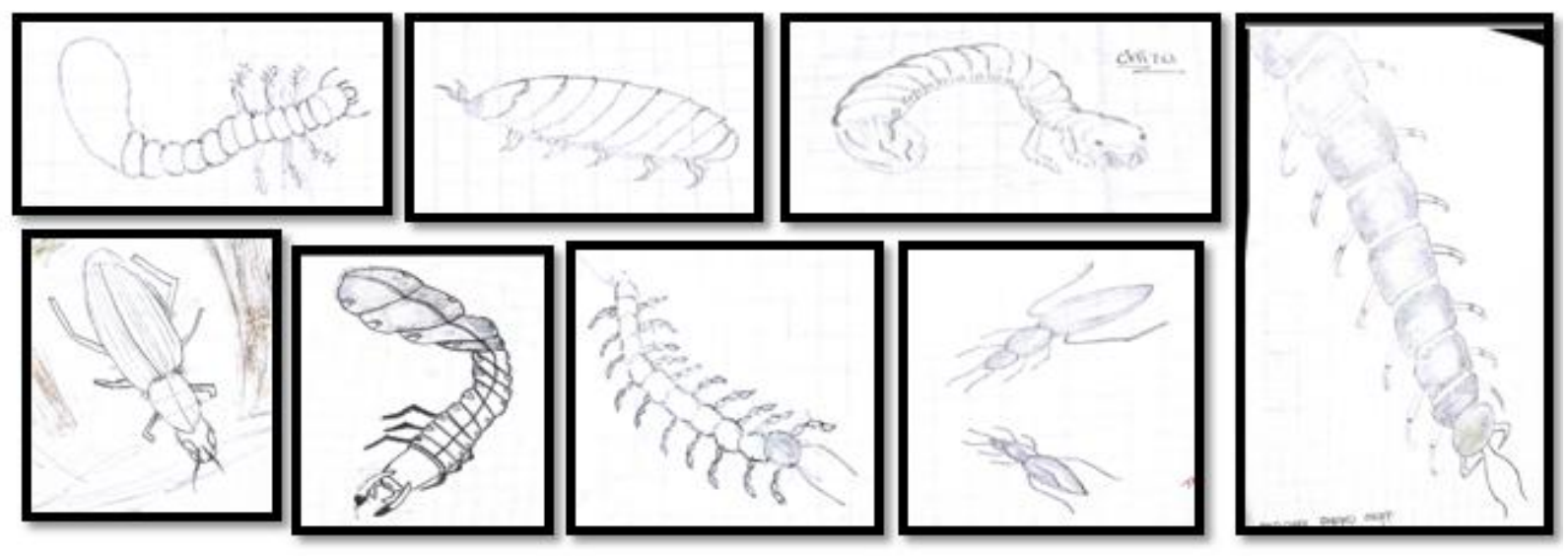

\section{La ilustración como un proceso de formación}

Desde habilidades como la observación se comienza a introducir una serie de elementos que van siendo procesados para emitir una respuesta y por tanto una asociación próxima al concepto grafico que se involucra.

A propósito del ejercicio que se realiza, es interesante comentar que dentro de las reflexiones desde la experiencia de los estudiantes se concuerda con que la relación del arte y la ciencia en el proceso de conocimiento a partir del detalle gráfico de la imagen, en 


\title{
Bio - grafía. Escritos sobre la Biología y su Enseñanza. ISSN 2027-1034 \\ Edición Extraordinaria. p.p. $1296-1313$
}

\author{
Memorias del IX Encuentro Nacional de Experiencias en Enseñanza de la Biología y la \\ Educación Ambiental. IV Congreso Nacional de Investigación en Enseñanza de la \\ Biología.
}

este sentido, se reflexiona frente a la sensibilidad artística que prueba en los rigores de la ciencia; el espíritu científico frente a las herramientas y sugestiones del arte, nombrando allí los gestores iniciales de difundir esta manifestación. (Arte y ciencia en el mismo dibujo,2004)

Así pues, la necesidad del humano por configurar una red de comunicación no verbal ha permitido que el desarrollo y aplicación de este campo artístico connote y significa más los procesos en los cuales se vincula con las ciencias experimentales, siendo así que por medio de la representación gráfica comprenda, describa su mundo circundante, ya que la ilustración científica a acompañado el proceso de surgimiento de las ciencias naturales, de allí se establecen claves que permiten identificar las funciones que explican las construcciones científicas que se fueron tejiendo en procesos importantes como expediciones botánicas que se hicieron en los siglos XVII y XIX, donde las organización por grupo visibilizaron naturalistas ilustrando su entorno y de ello como resultado al desarrollo de la sistemática, la botánica y Zoología. Por su parte, la ilustración anatómica para apreciar las estructuras y funcionalidad del cuerpo humano, la simbología como medio de comunicación que asiente en la construcción de este campo.

De esta manera, la ilustración científica en la formación de los estudiante en el campo de la Biología permite significar en el sujeto su realidad, desde la observación y esclarecimiento del detalle de lo que vislumbra ante él, pues el ejercicio configura una interpretación profunda de la realidad y de ello la forma en la que el sujeto quien ilustra la plasma en un papel, pues prueba rigores de la ciencia en la relación confluyente del arte con ella, en ese sentido, si bien, el arte en su gran magnitud abarca muchos elementos que resultan interesantes a trabajar, sin embargo, desde la representación inmediata de un concepto y la transcripción de lo observado. La implementación de la ilustración científica como importante en el desarrollo del campo de la investigación y en el proceso de enseñanza, aprendizaje, que se constituye a medida que se van emparentando las relaciones de comprensión y de compartir por parte de los actores allí inmersos, de los pares, tanto de estudiante y maestro como sujetos en contante formación y construcción de conocimientos además de ser un apoyo esencial para dicho desarrollo.

Siendo así, tomar la ilustración científica como elemento que se instaura en el ejercicio de la representación en el desarrollo artístico, con carácter técnico, permite acentuar en la ciencia biológica, la posibilidad de registrar el entorno natural y con ello contribuir, a su vez, a ampliar las capacidades del observar dentro y fuera de un contexto en donde se configura el sujeto junto con el desarrollo potencial de la sensibilidad estética. Pues, ello permite en el sujeto rescatar y resaltar la sensibilidad, el aprecio de lo que le rodea, y de cómo percibe y se sitúa en su lugar, esto sin llegar a decir que se mueva por una emoción netamente subjetiva de la realidad. Además, de otorgar y conceder un lenguaje que permite entender un poco más la complejidad de lo natural, hecho que además resalta el valor estético de la representación. Este valor estético se entiende como la provocación de expresiones frente a la realidad natural. 


\title{
Bio - grafía. Escritos sobre la Biología y su Enseñanza. ISSN 2027-1034 \\ Edición Extraordinaria. p.p. $1296-1313$
}

\author{
Memorias del IX Encuentro Nacional de Experiencias en Enseñanza de la Biología y la \\ Educación Ambiental. IV Congreso Nacional de Investigación en Enseñanza de la \\ Biología.
}

En esta perspectiva, comenzar a configurar una serie de elementos que fortalecen esa noción me permite relacionar e instaurar nuevas maneras de conformación de la enseñanza de la Biología de manera integral, desde el objeto de estudio de la Biodiversidad local, si bien correlacionar la ilustración científica, pues allí, se van desarrollando y fortaleciendo las habilidades que se van constituyendo en las interacciones, haciendo que la observación, la sensibilidad y el acercamiento frente a otro grupo de organismos, permita edificar el reconocimiento de la biodiversidad a partir de las relaciones que allí confluyen, ejercicio que resulta interesante por quien lo realiza y además logra significar.

\section{Ilustración científica en relación a la Enseñanza de la Biología}

La enseñanza de biología integra la teoría, pero no siempre el alumno puede adquirir la formación integral, con actividades prácticas; lo cual no promueve la curiosidad científica del alumno, y es desde allí que la practica mediante la sensibilización el ejercicio de realizar una ilustración supone una serie elementos que se infunden en la concentración y atención en los detalles que allí se ostentas para recrear y memorizar, pues como plantea Jeanette Collins "la ilustración, a diferencia de la pintura, siempre debe realizar una función concreta: siempre debe tener una razón para existir" y no sólo ello, sino también en ese ejercicio que lleva al sujeto a pasar por un proceso de sensibilización o de estética frente a lo que esta ilustrando pues ello resulta como apoyo esencial para el desarrollo del conocimiento además de involucrar la cognición del sujeto, de un sentido de sensibilización y consciencia, haciendo necesario que las estrategias de enseñanza trasciendan lo actitudinal, pues en palabras de Jastrzbski (1985) Dibujar es un proceso de pensamiento, especialmente cuando es utilizado para la explicación visual de la investigación científica. No solo se significa la acción de ilustrar un algo, sino se interpreta y analiza desde las apreciaciones de lo estético del sujeto a la hora de ejecutar la acción.

Desde la práctica pedagógica pensar en la apuesta desde lo estético permite reflexionar que en el sujeto surgen posibilidades de comprender las configuraciones de los fenómenos circundantes del medio natural, en este sentido, construye relaciones en los procesos cognitivos que allí se están generando, además que desde el licenciado en Biología permite pensar en la enseñanza de la Biología frente al arte por que permite dimensionar, mediante un lenguaje visual el desarrollo y fortalecimiento de habilidades científicas como la observación, descripción del detalle de los códigos que le son propios.

De esta manera, la ilustración científica es una rama de la ilustración artística y una herramienta fundamental en la comprensión del conocimiento. Estamos de acuerdo con Carlino (2005) cuando afirma que se aprende ciencias leyendo y escribiendo en ella; de manera análoga, se aprende ciencia también observando imágenes y realizándolas. Como hemos visto, la ciencia se construye desde lo verbal y desde lo gráfico.

Javier Grilli, Mirtha Laxague \& Lourdes Barboza se sintetiza la idea de la manifestación de la ilustración científica en el desarrollo de las ciencias, el dibujo en ciencias es arte, pero se diferencia del dibujo artístico en la correspondencia que debe tener con el mundo natural que pretende representar y describir. Se habla del papel fundamental en la 
Bio - grafía. Escritos sobre la Biología y su Enseñanza. ISSN 2027-1034

Edición Extraordinaria. p.p. $1296-1313$

Memorias del IX Encuentro Nacional de Experiencias en Enseñanza de la Biología y la

Educación Ambiental. IV Congreso Nacional de Investigación en Enseñanza de la Biología.

enseñanza y aprendizaje de las ciencias naturales que en este caso dirige la atención en la educación básica secundaria como elemento que posibilita el acercamiento y la aprehensión de las configuraciones que se dan en el medio natural.

No obstante, tejer todo un discurso frente a lo que se evidencia y se explora del sujeto, con un objeto claro de estudio en la Biología, me permite establecer una relación dependiente del arte solamente desde su expresión artística de la ilustración científica como el elemento por el cual se acercan a esas sensibilidades (desde lo estético) a la apreciación en torno a la configuración del sujeto en el territorio a partir de su interacción directa.

Estamos de acuerdo con Carlino (2005) cuando afirma que se aprende ciencias leyendo y escribiendo en ella; de manera análoga, se aprende ciencia también observando imágenes y realizándolas. Como hemos visto, la ciencia se construye desde lo verbal y desde lo gráfico.

La ilustración científica desde Coccusi (2000) se define desde el carácter exigente de la fidelidad con la naturaleza en el propósito de la representación grafica clara. Puesto que se resalta la importancia de ésta como la primera manifestación gráfica del hombre primitivo. La necesidad de los sistemas de enseñanza requirió la implementación de documentos gráficos. Menciona a su vez las características y elementos claves y únicos que los principios estéticos apoyen la composición y valoración de la ilustración que se considera. .

\section{Reflexiones...}

Desde el establecimiento y aplicación de la practica pedagógica ha permitido ir contextualizando la forma en que la escuela esta abordando el saber en las ciencias naturales, y de lo que es necesario para la formación de los estudiantes en términos de constante aprehensión, en este sentido, desde lo que se planteo inicialmente, la ilustración científica pensada desde su configuración (a propósito de la pregunta por el cómo) y organización como estrategia pedagógica permitiría en primero momento la innovación en términos de rescate y fomento de este campo, pues si bien, data desde el desarrollo próximo de las ciencias y por supuesto en el auge y desarrollo de la historia natural como saber.

Como se menciona a lo largo del documento, no es posible acercarse a la constitución de la ilustración científica en tanto estrategia pedagógica, sin embargo, con los ejercicios realizados, permite dejar una puerta de investigación abierta en términos de rescatar aquel saber que era de carácter imprescindible para el aprendizaje de las ciencias.

La pregunta por el porqué la ilustración científica debe configurase como una posible estrategia de enseñanza de la Biodiversidad (siendo el caso de la investigación) aunque abierto a toda posibilidad de estudio. Pues se optimiza de potencial porque permite construir e involucrar en la comunicación de la comunidad científica un lenguaje visual 


\title{
Bio - grafía. Escritos sobre la Biología y su Enseñanza. ISSN 2027-1034 \\ Edición Extraordinaria. p.p. $1296-1313$
}

\author{
Memorias del IX Encuentro Nacional de Experiencias en Enseñanza de la Biología y la \\ Educación Ambiental. IV Congreso Nacional de Investigación en Enseñanza de la \\ Biología.
}

que es fiel al entorno y por ende para fortalece aquellas habilidades científicas que el sujeto sustenta, que se han venido sesgando como proceso complementario en la formación, construcción y producción de conocimiento en las ciencias.

Pensar la ilustración científica como posibilidad de investigación, pues presenta varios pilares que fomentan en la investigación su uso desmesurado desde la comunicación hasta la innovación en el capo escolar, a propósito de la pertinencia curricular que actualmente se establece en la escuela. Es así, que desde su implemento y acercamiento al aula, puede ser constituida como saber en términos del aproximación y conocimiento, para toda el área de estudio de las ciencias naturales, en este caso de los artrópodos, no obstante de cómo se ve morfológicamente sino desde su rol y toda la cadena de interacciones que va a efectuar allí, para poder comenzar a entender este tipo de comportamientos, además de ello de consolidar una proximidad en el saber frente a la Biodiversidad.

Dentro del proceso de formación de los estudiantes, éste ejercicio plantearía de forma dinámica, pues involucra al contexto con el sujeto, permitiendo así conocer, comprender, analizar, discutir lo que pasa a su alrededor, además de comunicar un mensaje, pues lo que la imagen permite y evoca hace particular esta constante relación de conocimientos pues no solo se constituye en un saber meramente biológico (claro esta, sin desconocerlo) sino que también permite abarcar los procesos del sujeto en relación ese contacto que denota emociones, constructos propios idealistas frente a la realidad.

De su vinculación en la escuela, Lowenfeld (1986), viene planteando el arte como una válvula reguladora entre el intelecto y el sentir de un sujeto, pero hoy día esto no es tenido en cuenta, se está sometiendo el aprendizaje del niño al cumplimiento de estándares y logros que imposibilita un desarrollo integral y complejo, que lo ata a la reproducción y conexión constante a medios masivos, manteniéndose distante del entorno, de la naturaleza.

\section{CONCLUSIONES}

La generación de espacios de reflexión de la acción humana frente a cómo observa y comprende el mundo a través del trazo en la ilustración científica, crea significaciones simbólicas y figurativas en el proceso de enseñanza aprendizaje, además de configurarla como estrategia en el campo de acción. A partir de la ilustración científica, quien realiza el ejercicio de ilustrar, no solo se sitúa en las formas y colores que aprecia de su realidad, también genera en el sujeto la toma conciencia de los planos de la realidad en espacio y tiempo al realizar trazos, pues en el ejercicio de interpretación asocia la adquisición significativa de conocimientos.

Desde la practica pedagógica como escenario que posibilita la realización de ejercicios de investigación en espacios diversos, se concentra dentro del marco de la educación en la escuela, la necesidad de pensar cuál es el lugar y el valor que se atribuye a lo estético, 
Bio - grafía. Escritos sobre la Biología y su Enseñanza. ISSN 2027-1034

Edición Extraordinaria. p.p. $1296-1313$

Memorias del IX Encuentro Nacional de Experiencias en Enseñanza de la Biología y la

Educación Ambiental. IV Congreso Nacional de Investigación en Enseñanza de la Biología.

desde el sentido apreciativo. No obstante, es importante la constante reflexión en torno a la significación de la enseñanza de la Biología, desde el ejercicio de la ilustración científica como manifestación del arte desde la importancia de analizar y comprender las actitudes determinantes hacia el arte y la necesidad de entrar en contacto con sus manifestaciones, de la valoración y sensibilidad por el hombre frente lo que observa, y a su vez de la posibilidad de pensar lo sensible sin dejar de lado los parámetros que se establecen en el ejercicio de la ilustración científica.

Desde la formación como licenciados éste se consolida como un gran elemento que trasciednde su constructo, pues permite el desarrollo de nuevas habilidades y por lo tanto de construcción de cocnocmientos, pues, la ilustración científica se ha insertado como una de las formas más específicas de la comunicación visual en las ciencias. El objetivo de la ilustración es representar exactamente lo que es necesario. Un punto muy importante en la ilustración, es la cualidad de reafirmar visualmente caracteres diferenciados de los especímenes representados.

la ilustración es de carácter activo lo que implica que puede generar procesos de transformación frente a concepciones y percepciones de lo vivo, sin embargo es un cuerpo de estudio que aún no tiene fuerza suficiente para renacer.

\section{BIBLIOGRAFÍA}

- Asencio, M. (2001). Marco teórico del aprendizaje informal. EN Asencio, M., Pol, E. (Ed.), Nuevos escenarios en educación, aprendizaje informal sobre el patrimonio los museos y la ciudad. Buenos Aires, Editorial Aique

- Beltrán, J. (1993). Procesos, estrategias y técnicas de aprendizaje. Madrid: Síntesis

- Benndeto, A. (2006). Identidad y territorio: aportes para la re-valorización de los procesos de diferenciación productiva en áreas de co-existencia geográfica (Mendoza, Argentia).

- Calero, M. \& Conti, M. (2009). El diario de campo, una herramienta de investigación educativa utilizada en el aula multigrado. Quehacer educativo, 75-77.

- Cocucci, A. 2000. Dibujo cientifico. Manual para biológos que no son dibujantes y dibujantes que no son biológos. Cordoba: Sociedad Argentina de Botánica

- Di Castri F, Younès T (Eds.) (1996) Biodiversity, science and development. CAB International/IUBS. Paris, Francia. pp. 1-11.

- England E., Hinojosa D., Romero M. (2010) llustración científica en el IES Antonio. 
Bio - grafía. Escritos sobre la Biología y su Enseñanza. ISSN 2027-1034

Edición Extraordinaria. p.p. $1296-1313$

Memorias del IX Encuentro Nacional de Experiencias en Enseñanza de la Biología y la Educación Ambiental. IV Congreso Nacional de Investigación en Enseñanza de la Biología.

- Espinosa D, Cordero C (1995) Biodiversidad, instrumento para medir la vitalidad de la naturaleza. La Jornada Ecológica. Suplemento de La Jornada. México. 5/04/1995. Proyecto Curricular de la Licenciatura en Biología. Universidad Pedagógica Nacional.

- Hall, V. C., Bailey, J. y Tillman, C. (1997). Can student-generated illustrations be worth ten thousand words? Journal of Educational Psychology, 89(4), 677-681

- Ley 1549 de 2012; objetivos y estrategias de la política nacional de educación ambiental.

- Ley 115 de 1994, Art. 66 y 97.De Educación Ambiental

- Lowenffeld, V. 1986. El niño y su Arte. Buenos Aires : KAPELUSZ

- Márquez C. (2002) Dibujar en las clases de ciencia. Aula de Innovación Educativa 117, 54-57

- Morales, A. (octubre- diciembre de 2003). Los paradigmas de investigación en ciencias sociales. ISLAS, 45(138):125-135

- Manual de convivencia. Instituto Técnico Industrial Francisco José de Caldas. Agenda 2015

- Proyecto Curricular Licenciatura en Biología (2011) Universidad Pedagógica Nacional. Documento de referencia

- Sarmiento, E. (2014). Documento con la propuesta para la implementación del servicio social ambiental y las pasantías dentro del JBB y en la región capital con la orientación del JBB. . Bogotá.

- Sordo M, Verónica (2006). La importancia del desarrollo de las habilidades del proceso científico en el aprendizaje de la ciencia y su aplicación en proyectos de ciencia experimental para niños. Memoria de la segunda reunión nacional de análisis: La Actividad Experimental en el Aprendizaje de las Ciencias Naturales y Exactas. Culiacán, Sin. 29, 30 y 31 de marzo de 2006. 